\title{
Efeitos do flúor em folhas de plantas aquáticas de Salvinia auriculata
}

\author{
Paulo Henrique Pereira Peixoto(1), Daniel Sales Pimenta(1) e Flávia Antunes $^{(1)}$
}

\begin{abstract}
(1)Universidade Federal de Juiz de Fora, Instituto de Ciências Biológicas, Dep. de Botânica, CEP 36036-330 Juiz de Fora, MG. E-mail: phpp@icb.ufff.br, dsp@icb.ufjf.br, antunes.flavia@bol.com.br
\end{abstract}

\begin{abstract}
Resumo - O objetivo deste trabalho foi avaliar os efeitos do flúor em folhas de plantas aquáticas de Salvinia auriculata Aubl., visando fornecer subsídios para a utilização dessa espécie, no monitoramento da poluição ambiental, causada por esse elemento tóxico. As plantas foram cultivadas sob condições controladas, em vasos com solução nutritiva, e submetidas à aplicação de chuva simulada contendo KF, nas concentrações de $0,13,26$ e 39 mM, pela manhã, durante cinco dias sucessivos. Os resultados evidenciaram a ocorrência de alterações morfológicas, com o desenvolvimento de lesões nos tricomas e na porção adaxial do limbo foliar. As alterações nas atividades das enzimas peroxidase, polifenol oxidase, superóxido dismutase e catalase indicaram a ocorrência de danos oxidativos em resposta ao flúor, embora testes relacionados à peroxidação dos lipídios tenham apresentado resultados negativos. As alterações na concentração de pigmentos também direcionam para a ocorrência de estresse oxidativo, causado pelo flúor, presente na chuva simulada. Como as alterações morfológicas, enzimáticas e na composição de pigmentos, de plantas de S. auriculata, são passíveis de detecção por métodos relativamente simples, elas podem ser empregadas no biomonitoramento da poluição atmosférica, causada por esse elemento altamente reativo.
\end{abstract}

Termos para indexação: estresse oxidativo, bioindicadores, biomonitoramento.

\section{Fluoride effects on leaves of aquatic Salvinia auriculata}

\begin{abstract}
The objective of this work was to evaluate the effects of fluoride on leaves of aquatic Salvinia auriculata Aubl., to supply subsidies for the use of this species as an environmental pollution bioindicator. The plants were cultivated under controlled conditions in pots containing nutritive solutions, and were submitted to simulated rain containing KF, in concentrations of 0, 13, 26 and $39 \mathrm{mM}$, in the morning, during five consecutive days. Results evidenced the occurrence of morphological and anatomical damages involving trichome and adaxial surface of the leaf necrosis. Alterations in the activity of peroxidase, polyphenoloxidase, superoxide dismutase, and catalase enzymes indicated the occurrence of oxidative damages in response to fluoride, although lipid peroxidation tests showed negative results. Alterations in pigment concentration also pointed to the occurrence of oxidative stress, caused by fluoride present in the simulated rain. The morphological, anatomical, enzymatic, and pigment composition alterations in S. auriculata can be used in atmospheric pollution biomonitoring, because they are easy to detect through simple methodologies.
\end{abstract}

Index terms: oxidative stress, bioindicators, biomonitoring.

\section{Introdução}

A sensibilidade dos organismos, a diferentes substâncias tóxicas, é critério fundamental na escolha de bioindicadores de poluição (Lewis, 1995). Algumas espécies são particularmente sensíveis à poluição (bioindicadoras de resposta), enquanto outras, as bioindicadoras de acumulação, podem acumular poluentes em concentrações elevadas, sem apresentarem sintomas visíveis (Silva et al., 2000).

Diversas espécies de plantas aquáticas podem agir como “fitorremediadoras”, removendo resíduos sólidos em suspensão, nutrientes, metais pesados, bactérias e outras substâncias orgânicas tóxicas encontradas na água (Lewis, 1995). A Salvinia spp., uma macrófita livre e flutuante muito comum em água doce, sob condições favoráveis é rapidamente disseminada por propagação vegetativa, colonizando extensas superfícies de água em um tempo reduzido. As elevadas taxas de crescimento, e a sensibilidade das plantas de Salvinia a diferentes agentes tóxicos, justificam sua utilização como espécie “sentinela” e bioindicadora de poluição em ecossistemas aquáticos (Gardner \& Al-Hamdani, 1997). 
Espécies que são mais sensíveis ao flúor (F) apresentam lesões em resposta à exposição rápida (1 a 3 dias), a concentrações de HF menores que 1 ppb $\left(0,8 \mu \mathrm{g} \mathrm{m}^{-3}\right)$. Concentrações elevadas e exposição prolongada ao $\mathrm{F}$ causam danos rápidos e intensos (Weinstein \& Davison, 2003). Normalmente, a concentração de F na precipitação atmosférica varia entre 1 e $1000 \mu \mathrm{g} \mathrm{L}^{-1}$ e pode alcançar valores mais elevados, até $10 \mathrm{mg} \mathrm{L}^{-1}$, em áreas industriais (Smith \& Hodge, 1979). Em águas não poluídas, a concentração de $\mathrm{F}$ normalmente varia entre 0,01 e $0,3 \mathrm{mg} \mathrm{L}^{-1}$. Nas áreas próximas às siderúrgicas de alumínio, a concentração de F, proveniente da precipitação atmosférica, pode alcançar valores até $0,05 \mathrm{mg} \mathrm{L}^{-1}$. Nas proximidades das fábricas de fertilizantes fosfatados, a concentração desse poluente pode alcançar valores até 100 vezes mais elevados do que o normal (Camargo, 2003). Embora a detecção de F na atmosfera, por meio de métodos químicos, seja precisa, ela é muito cara (Arndt et al., 1995), o que justifica a utilização de plantas como bioindicadoras da poluição causada por esse elemento altamente reativo.

De um modo similar à maioria dos poluentes atmosféricos, o $\mathrm{F}$ penetra nas plantas através dos estômatos. Como conseqüência, sintomas crônicos tornam-se evidentes, como a murcha dos lóbulos e, em alguns casos, a ocorrência de clorose marginal e internerval. Danos mais intensos causados por $\mathrm{F}$ são o escurecimento rápido e a morte dos tecidos da folha (Arndt et al., 1995; Weinstein \& Davison, 2003).

Os efeitos do $F$ na vegetação não são limitados aos danos foliares ou à redução do crescimento e da produtividade vegetal; eles também podem resultar em problemas de saúde pública. O F, na forma de HF, pode ser incorporado à cadeia alimentar e causar "fluorose" em animais que se alimentam da vegetação contaminada (Weber et al., 1994). Estudos relacionados à ocorrência de poluição causada por F, na Cidade de Juiz de Fora, MG, são inexistentes. Todavia, os níveis de partículas em suspensão e de inalantes no ar, em diferentes áreas da cidade, são mais elevados do que em grandes centros urbanos industrializados (Bara, 2002).

O F pode afetar os sistemas enzimáticos e as membranas, alterar e interromper cadeias metabólicas que resultam em estresse oxidativo (Arndt et al., 1995). A sensibilidade das plantas aos danos oxidativos depende do equilíbrio entre os fatores que aumentam a geração de espécies reativas e os compostos celulares com capacidade antioxidante (Jiménez et al., 1998). As espécies reativas de oxigênio (EROs) encontram-se natural- mente presentes em plantas, em diferentes níveis, o que é resultado do metabolismo aeróbio. Sob condições normais, as plantas possuem mecanismos que mantêm as EROs em níveis reduzidos (Scandalios, 1993).

Apesar de o F ser considerado um importante poluente, uma vez que sua concentração em ecossistemas aquáticos é aumentada, significativamente, como conseqüência de atividades antropogênicas, pouco se conhece de seus efeitos sobre a vida aquática. Portanto, a implantação de programas de biomonitoramento depende do desenvolvimento de pesquisas prévias, relacionadas à avaliação das alterações morfológicas, fisiológicas, anatômicas e bioquímicas, entre outras, causadas pelo F, a fim de esclarecer os possíveis mecanismos de resistência ou suscetibilidade a esse poluente, em diferentes espécies vegetais (Silva et al., 2000).

O objetivo deste trabalho foi avaliar os efeitos de F, dissolvido em chuva simulada, na estrutura anatômica, na atividade de enzimas do metabolismo oxidativo, na peroxidação de lipídios e na composição de pigmentos hidrossolúveis e lipossolúveis de folhas de Salvinia auriculata.

\section{Material e Métodos}

Plantas aquáticas Salvinia auriculata Aubl., coletadas em uma represa em Juiz de Fora, MG, foram usadas nos experimentos, que foram conduzidos em sala de crescimento, com temperatura controlada $\left(25 \pm 3^{\circ} \mathrm{C}\right)$, sob radiação fotossinteticamente ativa de $250 \mu$ moles $\mathrm{m}^{-2} \mathrm{~s}^{-2}$ e fotoperíodo de 16 horas. As plantas foram cultivadas em vasos de polietileno, com solução nutritiva (Clark, 1975), 1/3 da força, pH 6 ajustado a cada dois dias. As plantas foram submetidas à aplicação de $50 \mathrm{~mL}$ de chuva simulada com F, pela manhã, durante cinco dias sucessivos. A chuva foi aplicada nas concentrações de 0, 13, 26 e $39 \mathrm{mM}$, utilizando-se o KF.

A evolução dos sintomas morfológicos da toxidez foliar, causados pelo F, foi monitorada diariamente. Para a análise da morfologia interna, foram coletados pequenos fragmentos de folhas, 14 dias após o término da aplicação da chuva simulada. Os fragmentos foram transferidos para solução de AFA 50\% (etanol, formol e ácido acético - 9:0,5:0,5; v/v), para paralisar as reações metabólicas. Após dois dias, o material foi transferido para etanol/água (7:3; v/v) (Johansen, 1940). Em seguida à obtenção dos cortes, os mesmos foram submetidos aos processos de coloração com fucsina básica e azul de astra (Roeser, 1972) e foram montados em 
glicerina-água 50\% (v/v). A análise do material foi feita em fotomicroscópio.

Os ensaios enzimáticos foram realizados a partir de tecidos de folhas coletadas 24, 48 e 96 horas, após o término da chuva simulada com F. O extrato enzimático bruto, usado para determinação das atividades enzimáticas, foi obtido pela homogeneização de $0,2 \mathrm{~g}$ de matéria fresca (MF) de folhas, em $5 \mathrm{~mL}$ de tampão fosfato de potássio 0,1 M, pH 6,8, com EDTA 0,1 mM (Kar \& Mishra, 1976). O homogenato foi filtrado através de quatro camadas de gaze e, então, centrifugado a $6.000 \mathrm{~g}$ por $30 \mathrm{~min}$ a $2 \pm 1^{\circ} \mathrm{C}$. O sobrenadante foi usado nos ensaios enzimáticos.

Para a determinação da atividade da peroxidase (POD), uma alíquota de $25 \mu \mathrm{L}$ de extrato enzimático bruto foi acrescentada a $5 \mathrm{~mL}$ de uma mistura de reação, constituída de tampão fosfato de potássio $25 \mathrm{mM}$, pH 6,8, pirogalol $20 \mathrm{mM} \mathrm{e}_{2} \mathrm{O}_{2} 20 \mathrm{mM}$ (Kar \& Mishra, 1976). A reação foi interrompida pela adição de $200 \mu \mathrm{L}$ de $\mathrm{H}_{2} \mathrm{SO}_{4} 5 \%$ (v/v), após incubação por $2 \mathrm{~min}$ a $25^{\circ} \mathrm{C}$. Então, as absorvâncias das amostras foram lidas a 420 nm (Kar \& Mishra, 1976). A atividade da POD foi calculada pela determinação da quantidade de purpurogalina formada, utilizando-se o coeficiente de extinção molar de 2,47 mM-1 $\mathrm{cm}^{-1}$ (Chance \& Maehly, 1955).

Para a determinação da atividade da polifenol oxidase (POL), uma alíquota de $50 \mu \mathrm{L}$ de extrato enzimático bruto foi acrescentada a $5 \mathrm{~mL}$ de uma mistura com o mesmo meio de reação, utilizado para a determinação da atividade da POD, exceção feita à exclusão do $\mathrm{H}_{2} \mathrm{O}_{2}$. A mistura foi incubada por $2 \mathrm{~min}$ a $25^{\circ} \mathrm{C}$, e a reação foi paralisada pela adição de $200 \mu \mathrm{L}$ de $\mathrm{H}_{2} \mathrm{SO}_{4} 5 \%$ (v/v) (Kar \& Mishra, 1976). A atividade da POL também foi calculada pela determinação da quantidade de purpurogalina formada, utilizando-se o coeficiente de extinção molar de $2,47 \mathrm{mM}^{-1} \mathrm{~cm}^{-1}$ (Chance \& Maehly, 1955).

A atividade da superóxido dismutase (SOD) foi determinada pela adição de $100 \mu \mathrm{L}$ de extrato enzimático bruto, a $3 \mathrm{~mL}$ de uma solução com L-metionina $13 \mathrm{mM}$, riboflavina $2 \mu \mathrm{M}$, cloreto azul de p-nitro-tetrazólio (NBT) $75 \mu \mathrm{M}$, e EDTA $100 \mathrm{nM}$, em tampão fosfato de sódio 50 mM, pH 7,8 (Del Longo et al., 1993). A reação aconteceu em uma câmara, sob a iluminação de uma lâmpada fluorescente de $15 \mathrm{~W}$, mantida dentro de uma caixa coberta com papel alumínio, a $25^{\circ} \mathrm{C}$. A reação, iniciada pela ligação da lâmpada, foi interrompida um minuto depois pelo desligamento da mesma. A atividade da SOD foi medida pela diferença no aumento da absorvância das amostras a $560 \mathrm{~nm}$, subtraído de um "branco" ao qual o extrato enzimático bruto não foi adicionado. Uma unidade de SOD foi definida como a quantidade de enzima necessária para inibir, em 50\%, a fotorredução do NBT (Giannopolitis \& Ries, 1977).

A atividade da catalase (CAT) foi determinada pela adição de $100 \mu \mathrm{L}$ de extrato enzimático bruto a $3 \mathrm{~mL}$ de uma solução com $\mathrm{H}_{2} \mathrm{O}_{2}$ 12,5 mM em tampão fosfato de potássio $50 \mathrm{mM}, \mathrm{pH} 7$, a $30^{\circ} \mathrm{C}$ (Havir \& McHale, 1987). A atividade de CAT foi determinada pela medição da redução na absorvância das amostras a 240 nm, em conseqüência do consumo de $\mathrm{H}_{2} \mathrm{O}_{2}$, usando-se o coeficiente de extinção molar de $36 \mathrm{M}^{-1} \mathrm{~cm}^{-1}$ (Anderson et al., 1995).

A peroxidação dos lipídios foi determinada de acordo com a metodologia proposta por Cakmak \& Horst (1991). Amostras de matéria fresca, de folhas com peso de $0,2 \mathrm{~g}$ foram homogeneizadas em $4 \mathrm{~mL}$ de ácido tricloroacético (TCA) 1\% (p/v). O homogenato foi filtrado através de quatro camadas de gaze e, em seguida, centrifugado a $6.000 \mathrm{~g}$ por $15 \mathrm{~min}$. Então, 0,5 mL do sobrenadante foi acrescentado a $1,5 \mathrm{~mL}$ de ácido tiobarbitúrico (TBA) 0,5\% (p/v) em TCA 20\% (p/v). Os tubos foram fechados e incubados sob agitação, em banho maria, a $95^{\circ} \mathrm{C}$ por 2 horas. A reação foi paralisada pela transferência dos tubos de reação para um banho de gelo. Posteriormente, as amostras foram centrifugadas a $6.000 \mathrm{~g}$ por $10 \mathrm{~min}$. A absorvância do sobrenadante foi lida a $532 \mathrm{~nm}$ e $660 \mathrm{~nm}$. A concentração do complexo aldeído malônico-ácido tiobarbitúrico (MDA-TBA) produzido foi calculada, utilizando-se o coeficiente de extinção molar de $155 \mathrm{mM}^{-1} \mathrm{~cm}^{-1}$ (Heath \& Packer, 1968). A produção de aldeídos voláteis foi determinada pela metodologia desenvolvida por Santos et al. (1993).

Para extração dos aldeídos, 0,05 g de tecidos foliares foi transferido para tubos rosqueáveis, com $10 \mathrm{~mL}$ de etanol $2 \%(\mathrm{v} / \mathrm{v})$. Os tubos foram fechados e agitados a $25^{\circ} \mathrm{C}$ por 2 horas. Alíquotas de $0,5 \mathrm{~mL}$ foram transferidas para tubos com 0,5 mL de 3-metil-2-benzotiazolinone hidrazona (MBTH) 0,1\% (p/v), seguido pela adição de $1,3 \mathrm{~mL}$ de $\mathrm{FeCl}_{3} \cdot 6 \mathrm{H}_{2} \mathrm{O}$ 0,23\% (p/v). Após 2 min de incubação, $3 \mathrm{~mL}$ de acetona foram adicionados aos tubos. Após agitação dos mesmos, a absorvância das amostras foi lida a $635 \mathrm{~nm}$ (Harman et al., 1982). A quantidade de aldeídos voláteis liberados foi expressa em termos de massa de formaldeído, substância utilizada como padrão numa curva de calibração (Reis et al., 1989). 
As concentrações de clorofila $a$ e $b$ e de carotenóides totais foram determinadas pela metodologia de Lichtenthaler (1987). Amostras de matéria fresca de folhas com peso de $0,2 \mathrm{~g}$ foram coletadas e submetidas à extração, em $5 \mathrm{~mL}$ de acetona $80 \%(\mathrm{v} / \mathrm{v})$ durante 24 horas, sob refrigeração. Os extratos com os pigmentos cloroplastídicos foram filtrados, e as amostras foram submetidas à leitura em espectrofotômetro.

A concentração de antocianinas foi determinada após a adição de amostras de folhas de $0,2 \mathrm{~g}$ a $5 \mathrm{~mL}$ de solução de extração, com metanol-HCl 1\% (v/v), sob refrigeração, por 24 horas (Downs \& Siegelman, 1963). Após filtração e centrifugação a $1.500 \mathrm{~g}$ por $10 \mathrm{~min}$, as amostras foram submetidas à leitura em espectrofotômetro, de acordo com a metodologia proposta por Mancinelli (1990).

O experimento foi conduzido em delineamento inteiramente casualizado, com cinco repetições por tratamento. As médias foram comparadas pelo teste de Tukey a $5 \%$ de probabilidade.

\section{Resultados e Discussão}

As análises morfológicas das plantas tratadas com $\mathrm{F}$ evidenciaram a ocorrência de áreas necróticas na superfície foliar, com formato arredondado e com tonalidade marrom escura, cuja intensidade foi diretamente proporcional à concentração de F utilizada; foi, também, observada nas plantas a ocorrência de danos parciais aos tricomas, que se tornaram enrugados e com tonalidade marrom escura, semelhante à observada nas áreas necróticas da lâmina foliar. Cortes transversais, realizados em áreas afetadas por F, também evidenciaram a ocorrência de danos parciais aos tricomas, tendo restado de sua estrutura característica apenas a base. A deterioração da epiderme adaxial e da porção superior do mesófilo também foi observada. Todavia, a porção inferior do mesófilo, formada por grandes câmaras de aerênquimas, foi preservada e permitiu a manutenção do sistema de flutuação.

Danos similares aos observados no presente estudo são freqüentemente relatados em diferentes tipos de vegetação expostos às emissões de $\mathrm{F}$, tanto em áreas industriais, quanto sob condições controladas (Silva et al., 2000; Fornasiero, 2001). Nas concentrações de F do experimento, as análises morfológicas demonstraram que plantas de Salvinia auriculata podem ser utilizadas como indicadoras da poluição, causada pelo $\mathrm{F}$, em ecossistemas aquáticos, principalmente, pela sensibili- dade dessa espécie, que apresenta danos de fácil visualização e quantificação, pois as lesões são diretamente proporcionais à concentração de $\mathrm{F}$ aplicada.

Nas primeiras 24 horas, após o término da aplicação da chuva simulada com $\mathrm{F}$, a atividade da peroxidase (POD) aumentou, significativamente, somente na concentração de 39 mM, e nenhuma diferença significativa na atividade dessa enzima foi observada nas demais concentrações (Figura 1). A atividade da POD, 48 horas após o término da chuva simulada com $\mathrm{F}$, mostrou, em relação ao controle, aumentos significativos nos diferentes tratamentos. Entretanto, nenhuma diferença significativa foi detectada entre as concentrações utilizadas. A tendência de aumento, na atividade da POD, foi mantida na análise realizada 96 horas após o término da chuva simulada. Nessa análise, foram observados aumentos significativos e diretamente proporcionais às concentrações de F utilizadas (Figura 1). De acordo com Siegel (1993), a atividade da POD é freqüentemente aumentada em resposta aos estresses, pois a proteção celular contra reações oxidativas é uma das principais funções dessa enzima. O aumento da atividade das PODs é uma resposta metabólica relacionada a diferentes tipos de estresses (Cakmak \& Horst, 1991; Anderson et al., 1995; Zhang \& Kirkham, 1996; Jiménez et al., 1998; Moraes et al., 2002).

A atividade da polifenol oxidase (POL) mostrou um comportamento similar ao observado para a POD (Figura 1). Nas concentrações de F de 26 mM e 39 mM, a atividade da POL aumentou significativamente, em relação ao controle, 24 horas após o término da chuva simulada, sem mostrar diferença estatística entre as médias. Contudo, 48 horas após o término da chuva simulada, a atividade da POL aumentou na concentração de $\mathrm{F}$ de $39 \mathrm{mM}$, sem diferenças significativas nas demais concentrações. A tendência de aumento na atividade da POL foi mantida na análise realizada, 96 horas após o término da chuva simulada, e o aumento foi diretamente proporcional à concentração de $\mathrm{F}$ utilizada.

A atividade da POL tem sido utilizada como parâmetro de avaliação da peroxidação de lipídios, e da produção de compostos fenólicos (Mayer, 1987). O acúmulo de compostos fenólicos e o aumento na atividade da POL podem gerar substâncias reativas, tais como as quinonas derivadas do ácido caféico que, juntamente com outros compostos fenólicos, são ativas na produção do superóxido $\left(\mathrm{O}_{2}-\right)$ e, conseqüentemente, na peroxidação de lipídios (Marschner, 1995). 
A atividade da superóxido dismutase (SOD) aumentou, significativamente, em relação ao controle, 24 horas após o término da chuva simulada com $\mathrm{F}$, independentemente da concentração usada (Figura 1). Esse comportamento foi observado na análise realizada, 48 horas após o término da chuva simulada com F, apenas na concentração de 39 mM. Contudo, a análise realizada 96 horas após o término da chuva simulada, novamente, evidenciou que a atividade da SOD, na presença de F, sofreu aumentos significativos em relação ao controle, principalmente na concentração de 39 mM.

Os aumentos observados nas atividades da POD, POL e SOD indicam que o F apresenta ação direta so- bre o metabolismo oxidativo das plantas, e causa a formação de $\mathrm{H}_{2} \mathrm{O}_{2}$ e do radical superóxido $\left(\mathrm{O}_{2}-\right)$, principais substratos dessas enzimas. Os radicais $\mathrm{O}_{2}-$ e os seus derivados, o radical hidroxila $(\mathrm{OH} \cdot)$ e oxigênio "singleto" $\left({ }^{1} \mathrm{O}_{2}\right)$, são as principais fontes de danos peroxidativos aos componentes celulares (Scandalios, 1993). O radical $\mathrm{O}_{2}$ - transforma-se, espontaneamente, em $\mathrm{H}_{2} \mathrm{O}_{2}$. Todavia, a reação é muito mais rápida, se catalisada pela SOD. A ação combinada da SOD e da CAT reduz o risco da formação de $\mathrm{OH}$ (radical hidroxila), um radical livre de elevada reatividade (Scandalios, 1993). Contudo, o $\mathrm{H}_{2} \mathrm{O}_{2}$ produzido representa uma outra espécie química, potencialmente tóxi-
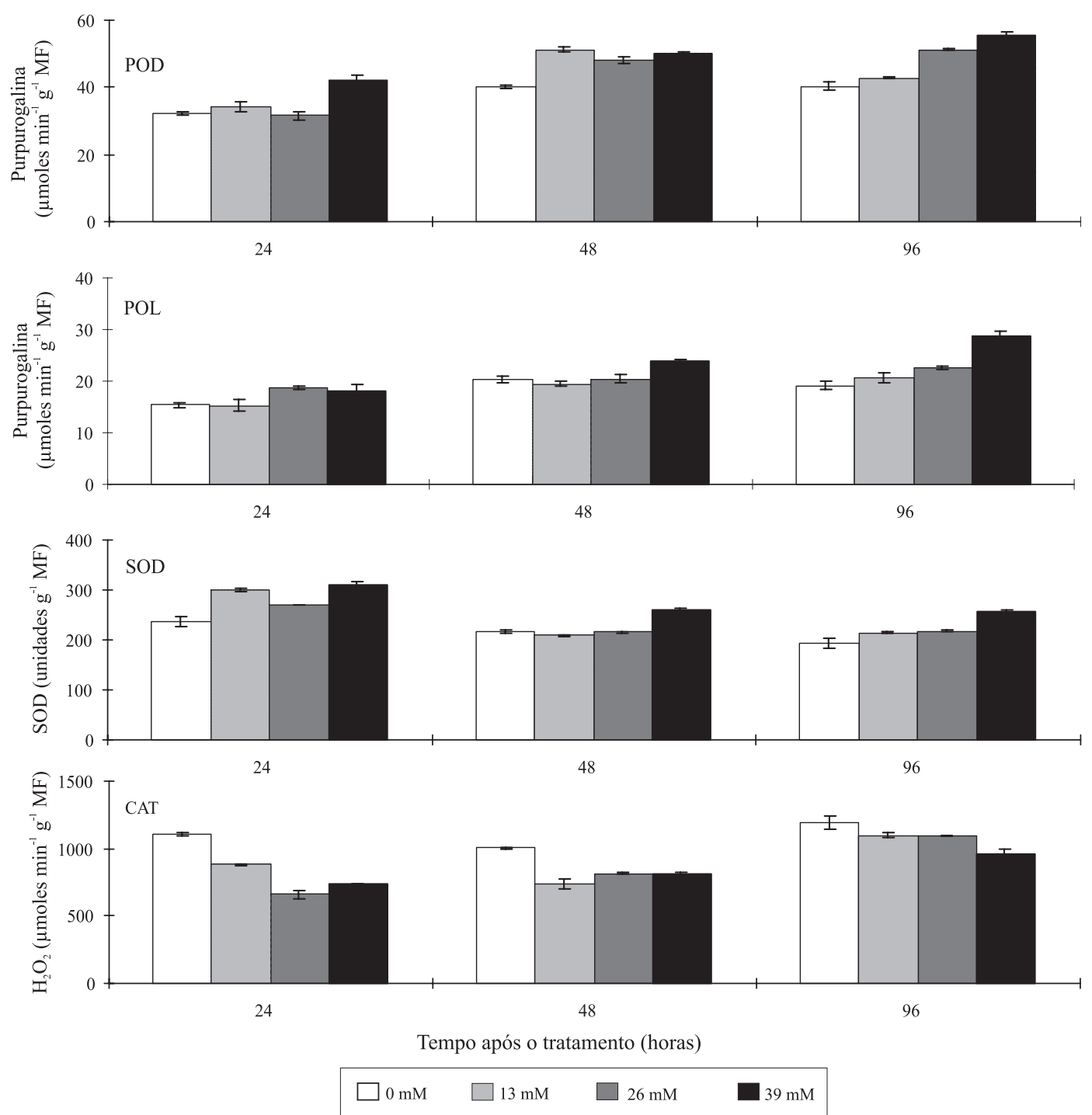

Figura 1. Atividade das enzimas peroxidase (POD), polifenol oxidase (POL), superóxido dismutase (SOD) e catalase (CAT ) na matéria fresca (MF) de folhas de Salvinia auriculata, submetidas ao tratamento com flúor. As barras em cada coluna representam as médias \pm erro-padrão (n=5). 
ca, devendo ser rapidamente eliminada do metabolismo (Foyer et al., 1994). A ação combinada da SOD, da CAT e de diferentes PODs, além da atuação de substâncias antioxidantes de baixo peso molecular como o ascorbato pode, efetivamente, eliminar, varrer e imobilizar espécies tóxicas de oxigênio (Scandalios, 1993; Siegel, 1993).

A atividade da catalase (CAT), em resposta ao F, apresentou um comportamento distinto do observado para POD, POL e SOD. A atividade da CAT se reduziu, significativamente, na análise realizada 24 horas após o término da chuva simulada com F, principalmente nas concentrações de $26 \mathrm{mM}$ e $39 \mathrm{mM}$ (Figura 1). A tendência de redução na atividade da CAT foi mantida na análise realizada 48 horas após o término da chuva simulada, embora sem diferenças significativas entre as concentrações de F utilizadas. Esse comportamento também foi observado na análise, realizada 96 horas após o término da chuva simulada, com a redução na atividade da CAT, principalmente na concentração de 39 mM. De acordo com Zhang \& Kirkham (1996), a capacidade de manutenção, em níveis elevados, da atividade da CAT, da ascorbato peroxidase (APX) e da SOD, sob condições de estresses ambientais, é essencial para a manutenção do equilíbrio entre a formação e a remoção do $\mathrm{H}_{2} \mathrm{O}_{2}$ do ambiente intracelular. Segundo Cakmak \& Horst (1991), a redução na atividade da CAT e o aumento nas atividades da POD e da POL indicam que, em plantas mantidas sob condições de estresse, o $\mathrm{H}_{2} \mathrm{O}_{2}$ gerado é mais consumido em processos oxidativos, como na peroxidação de lipídios, do que eliminado do metabolismo.

Os aumentos nas atividades da POD, POL e SOD evidenciam que o metabolismo oxidativo das plantas de Salvinia auriculata foi ativado em resposta à ação tóxica do F. A ativação de metabolismo oxidativo nas plantas, submetidas à chuva simulada com $\mathrm{F}$, parece resultar na acumulação de $\mathrm{H}_{2} \mathrm{O}_{2}$ e, por conseguinte, na peroxidação de lipídios. De acordo com Buege \& Aust (1978), a peroxidação de lipídios tem início com a remoção de um átomo de hidrogênio das moléculas de ácidos graxos insaturados. O subseqüente rearranjo das duplas ligações resulta na formação de um dieno-conjugado que, após o ataque por oxigênio molecular, produz os radicais peroxilipídicos e os endoperóxidos (Gutteridge \& Halliwell, 1990). A formação de endoperóxidos, a partir de ácidos graxos insaturados, que apresentam pelo menos três metilenos interrompidos por ligações duplas, pode resultar na formação de dialdeído malônico (MDA), um produto da degradação dos lipídios (Buege \& Aust, 1978).
Os dois testes que poderiam confirmar a ocorrência da peroxidação de lipídios, nos tecidos tratados com $\mathrm{F}$, apresentaram resultados negativos. O conteúdo de aldeído malônico-ácido tiobarbitúrico (MDA-TBA), um indicativo da peroxidação dos lipídios, não foi alterado significativamente pelo aumento da concentração de $\mathrm{F}$ na chuva simulada (Tabela 1). Os resultados obtidos, no ensaio conduzido para detecção da produção de aldeídos voláteis, com a utilização do 3-metil-2-benzotiazolinona hidrazona (MBTH), também não indicaram diferenças significativas na produção dessas substâncias, que são diretamente relacionadas aos estresses oxidativos (Tabela 1). Os resultados encontrados nesses dois ensaios sugerem que o F, nas concentrações utilizadas, não interfere na produção de aldeído malônico, bem como na liberação de aldeídos voláteis e, conseqüentemente, na peroxidação dos lipídios. Ambos os testes, todavia, podem não ser suficientemente sensíveis para detectar a produção dessas substâncias, em folhas de $S$. auriculata submetidas ao estresse com $\mathrm{F}$.

Sakihama \& Yamasaki (2002) relataram, recentemente, que o principal produto de decomposição decorrente da peroxidação de lipídios, em plantas submetidas ao alumínio (Al), foi o 4-hidroxi-nonenal (4-HNE), e não substâncias reativas com o ácido tiobarbitúrico (TBA). Se algum outro teste for capaz de detectar a peroxidação de lipídios, em tecidos de $S$. auriculata submetidas ao estresse com F, na mesma faixa de concentração avaliada no presente experimento, a utilização dos ensaios do MDA-TBA e do MBTH deverá ser desaconselhada, uma vez que os resultados não serão parâmetros bioquímicos seguros para o monitoramento da poluição atmosférica causada pelo $\mathrm{F}$ em plantas dessa espécie.

As concentrações de clorofila $a$, clorofila $b$ e de carotenóides totais foram reduzidas, em resposta ao

Tabela 1. Concentração do complexo MDA-TBAe de aldeídos voláteis, em folhas (matéria fresca - MF) de Salvinia auriculata coletadas 24, 48, 72 e 144 horas após o término da chuva simulada, com diferentes concentrações de flúor ${ }^{(1)}$.

\begin{tabular}{ccccc}
\hline Concentração de flúor $(\mathrm{mM})$ & $24 \mathrm{~h}$ & $48 \mathrm{~h}$ & $72 \mathrm{~h}$ & $144 \mathrm{~h}$ \\
\hline \multicolumn{5}{c}{ MDA-TBA $($ nmoles de MDA-TBA g } \\
\hline 0 & $65,419 \mathrm{a}$ & $63,311 \mathrm{ab})$ & $56,000 \mathrm{a}$ & $74,838 \mathrm{a}$ \\
13 & $64,516 \mathrm{a}$ & $63,483 \mathrm{a}$ & $56,645 \mathrm{a}$ & $68,516 \mathrm{a}$ \\
26 & $63,483 \mathrm{a}$ & $57,161 \mathrm{~b}$ & $56,258 \mathrm{a}$ & $67,741 \mathrm{a}$ \\
39 & $63,225 \mathrm{a}$ & $61,548 \mathrm{ab}$ & $53,419 \mathrm{a}$ & $70,064 \mathrm{a}$ \\
\hline \multicolumn{5}{c}{ Aldeídos voláteis $\left(\mu \mathrm{g}\right.$ de formaldeído $\left.\mathrm{g}^{-1} \mathrm{MF}\right)$} \\
13 & $25,770 \mathrm{a}$ & $21,788 \mathrm{a}$ & $30,678 \mathrm{a}$ & $24,860 \mathrm{a}$ \\
26 & $25,108 \mathrm{a}$ & $23,338 \mathrm{a}$ & $31,040 \mathrm{a}$ & $25,036 \mathrm{a}$ \\
39 & $25,362 \mathrm{a}$ & $23,185 \mathrm{a}$ & $29,174 \mathrm{~b}$ & $25,280 \mathrm{a}$ \\
& $25,661 \mathrm{a}$ & $22,771 \mathrm{a}$ & $28,341 \mathrm{~b}$ & $25,124 \mathrm{a}$ \\
\hline
\end{tabular}

(1)As médias seguidas pela mesma letra, nas colunas, não diferem entre si pelo teste de Tukey a 5\% de probabilidade. 
aumento da concentração de flúor aplicado na chuva simulada (Figura 2). Em contraste, a concentração de antocianinas aumentou, em resposta ao incremento na concentração de F. Comportamento similar foi observado por Gardner \& Al-Hamdani (1997) em Salvinia, na presença de alumínio, e por Wilson \& Al-Hamdani (1997) em Azolla caroliniana, na presença de cromo. De acordo com esses autores, a redução na concentração dos pigmentos fotossintéticos pode ser resultante de danos à estrutura dos cloroplastos, bem como do aumento da atividade de clorofilases. O aumento da concentração de antocianinas, em plantas aquáticas submetidas a diferentes tipos de estresses, é relatado na literatura (Doong et al., 1993). Aparentemente, o F também interfere na biossíntese ou na atividade de enzimas relacionadas à biossíntese ou à degradação de pigmentos hidrossolúveis e lipossolúveis, em folhas de S. auriculata. Alterações na composição de pigmentos resultam em efeitos diretos sobre a fotossíntese e, conseqüentemente, sobre a produtividade das plantas.

Os resultados encontrados no presente trabalho evidenciaram que o F, aplicado na forma de chuva simulada, causa danos morfológicos em folhas de Salvinia auriculata. Aparentemente, esses danos são causados pelo aumento na produção de espécies reativas de oxigênio, em resposta ao acúmulo de F nos tecidos foliares. Isto é sugerido pelas alterações observadas na atividade enzimática e na concentração de pigmentos foliares.

As alterações morfológicas, enzimáticas e na composição de pigmentos, em tecidos foliares de Salvinia

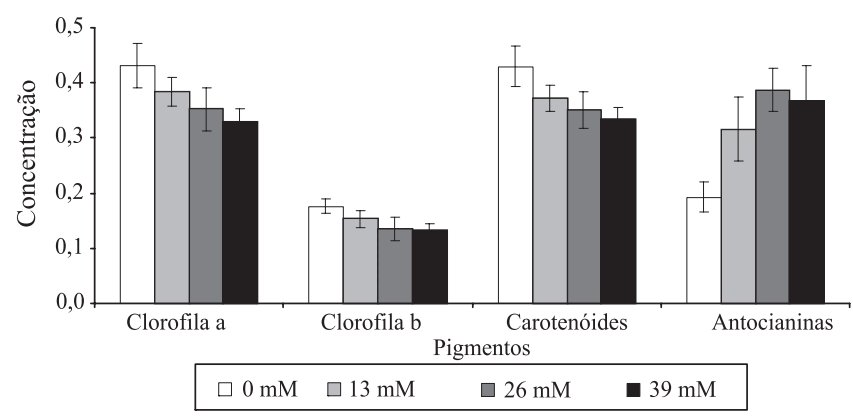

Figura 2. Efeitos do flúor na composição dos pigmentos, extraídos da matéria fresca (MF) de folhas de Salvinia auriculata, clorofila $a\left(\mathrm{mg} \mathrm{g}^{-1} \mathrm{MF}\right)$, clorofila $b\left(\mathrm{mg} \mathrm{g}^{-1} \mathrm{MF}\right)$, carotenóides totais $\left(100 \mathrm{x} \mathrm{mg} \mathrm{g}^{-1} \mathrm{MF}\right)$ e antocianinas $\left(10 \mathrm{x} \mu\right.$ moles $\left.\mathrm{g}^{-1} \mathrm{MF}\right)$. As barras em cada coluna representam as médias \pm erro-padrão (n=5). auriculata, são fáceis de serem detectadas, por meio de metodologias relativamente simples; por isso, tais alterações podem ser utilizadas no biomonitoramento da poluição atmosférica causada por esse elemento altamente reativo. Embora os dois testes para peroxidação de lipídios tenham apresentado resultados negativos, a possibilidade de que reações oxidativas, em resposta ao F, tenham ocorrido nos tecidos foliares das plantas de Salvinia auriculata não pode ser descartada.

\section{Conclusão}

As alterações morfológicas, enzimáticas e na composição de pigmentos em Salvinia auriculata podem ser utilizadas no biomonitoramento da poluição atmosférica causada pelo flúor.

\section{Agradecimentos}

Aos pesquisadores responsáveis pelos Laboratórios de Imunologia e Ecologia Aquática da UFJF, pelo apoio logístico.

\section{Referências}

ANDERSON, M.D.; PRASAD, T.K.; STEWART, C.R. Changes in isozyme profiles of catalase, peroxidase, and glutathione reductase during acclimation to chilling in mesocotylus of maize seedlings. Plant Physiology, v.109, p.1247-1257, 1995.

ARNDT, U.; FLORES, F.; WEINSTEIN, L. Efeitos do flúor sobre as plantas: diagnose de danos na vegetação do Brasil. Porto Alegre: Ed. da UFRGS, 1995. 155p.

BARA, G. Saúde em risco: poluição do ar atinge níveis críticos em Juiz de Fora. Tribuna de Minas, Juiz de Fora, 3 fev. 2002. Caderno 3, p.3.

BUEGE, J.A.; AUST, S.D. Microsomal lipid peroxidation. Methods in Enzymology, v.52, p.302-310, 1978.

CAKMAK, I.; HORST, W.J. Effect of aluminum on lipid peroxidation, superoxide dismutase, catalase, and peroxidase activities in root tips of soybean (Glycine max). Physiologia Plantarum, v.83, p.463-468, 1991.

CAMARGO, J. Fluoride toxicity to aquatic organisms: a review. Chemosphere, v.50, p.251-264, 2003.

CHANCE, B.; MAEHLY, A.C. Assay of catalases and peroxidases. Methods in Enzymology, v.2, p.764-775, 1955.

CLARK, R.B. Characterization of phosphatase of intact maize roots. Journal of Agriculture and Food Chemistry, v.23, p.458-460, 1975. 
DEL LONGO, O.T.; GONZÁLES, C.A.; PASTORI, G.M.; TRIPPI, V.S. Antioxidant defenses under hyperoxygenic and hyperosmotic conditions in leaves of two lines of maize with differential sensitivity to drought. Plant Cell Physiology, v.34, p.1023-1028, 1993.

DOONG, R.L.; MACDONALD, G.E.; SHILLING, D.G. Effect of fluridone on chlorophyll, carotenoid, and anthocyanin content of Hydrilla. Journal of Aquatic Plant Management, v.31, p.55-59, 1993.

DOWNS, R.J.; SIEGELMAN, H.W. Photocontrol of anthocyanin synthesis in milo seedlings. Plant Physiology, v.38, p.25-30, 1963.

FORNASIERO, R.B. Phytotoxic effects of fluoride. Plant Science, v.161, p.979-985, 2001.

FOYER, C.H.; LELANDAIS, M.; KUNERT, K.J. Photooxidative stress in plants. Physiologia Plantarum, v.92, p.696-717, 1994.

GARDNER, J.L.; AL-HAMDANI, S.H. Interactive effects of aluminum and humic substances on salvinia. Journal of Aquatic Plant Management, v.35, p.30-34, 1997.

GIANNOPOLITIS, C.N.; RIES, S.K. Superoxide dismutases: I- Occurrence in higher plants. Physiologia Plantarum, v.59, p.309-314, 1977.

GUTTERIDGE, J.M.C.; HALLIWELL, B. The measurement and mechanism of lipid peroxidation in biological systems. Trends in Biochemical Sciences, v.15, p.129-135, 1990.

HARMAN, G.E.; NEDROW, B.L.; CLARK, B.E.; MATTICK, R. Association of volatile aldehyde production during germination with poor soybean and pea seed quality. Crop Science, v.22, p.712-716, 1982.

HAVIR, E.A.; MCHALE, N.A. Biochemical and developmental characterization of multiple forms of catalase in tobacco leaves. Physiologia Plantarum, v.84, p.450-455, 1987.

HEATH, R.L.; PACKER, L. Photoperoxidation in isolated chloroplast: I- Kinetics and stoichometry of fatty acid peroxidation. Archives of Biochemistry and Biophysics, v.125, p.189-198, 1968.

JIMÉNEZ, A.; HERNÁNDEZ, J.A.; PASTORI, G.; DEL RIO, L.A.; SEVILLA, F. Role of ascorbate-glutathione cycle of mitochondria and peroxisomes in the senescence of pea leaves. Plant Physiology, v.118, p.1327-1335, 1998.

JOHANSEN, D.A. Plant microtechnique. New York: McGraw Hill, 1940. 523p.

KAR, M.; MISHRA, D. Catalase, peroxidase, and polyphenoloxidase activities during rice leaf senescence. Physiologia Plantarum, v.57, p.315-319, 1976.

LEWIS, M.A. Use of freshwater plants for phytotoxicity testing: a review. Environmental Pollution, v.87, p.319-336, 1995.

LICHTENTHALER, H.K. Chlorophylls and carotenoids: pigments of photosynthetic biomembranes. Methods in Enzymology, v.148, p.350-382, 1987.
MANCINELLI, A.L. Interaction between light quality and light quantity in photoregulation of anthocyanin production. Plant Physiology, v.92, p.1191-1195, 1990.

MARSCHNER, H. Mineral nutrition of higher plants. $2^{\text {nd }}$ ed. London: Academic Press, 1995. 889p.

MAYER, A.M. Polyphenol oxidases in plants: recent progress. Phytochemistry, v.26, p.11-20, 1987.

MORAES, R.M.; KLUMP, A.; FURLAN, C.M.; KLUMP, G.; DOMINGOS, M.; RINALDI, M.C.S.; MODESTO, I.F. Tropical fruit trees as bioindicator of industrial air pollution in Southeast Brazil. Environment International, v.28, p.367-374, 2002.

REIS, W.J.P.; ROCHA, V.S.; REZENDE, S.T.; MOREIRA, M.A.; SEDIYAMA, C.S. Correlação entre a evolução de n-hexanal e aldeídos totais e a germinação e vigor de sementes de soja. Revista Ceres, v.36, p.27-37, 1989.

ROESER, K.R. Die nadel der schwarziefer-massenprodukt und kunstwerk der natur. Mikrokosmos, v.61, p.33-36, 1972.

SAKIHAMA, Y.; YAMASAKI, H. Lipid peroxidation induced by phenolics in conjunction with aluminum ions. Biologia Plantarum, v.45, p.249-254, 2002.

SANTOS, I.C.; REIS, W.J.P.; MOREIRA, M.A.; REZENDE, S.T.; ROCHA, V.S.; SEDIYAMA, C.S. Determinação de aldeídos totais para avaliar o potencial de germinação de sementes de soja. Revista Ceres, v.40, p.438-444, 1993.

SCANDALIOS, J.G. Oxygen stress and superoxide dismutases. Plant Physiology, v.101, p.7-12, 1993.

SIEGEL, B.Z. Plant peroxidase: an organismic perspective. Plant Growth Regulation, v.12, p.303-312, 1993.

SILVA, L.C.; AZEVEDO, A.A.; SILVA, E.A.; OLIVA, M.A. Flúor em chuva simulada: sintomatologia e efeitos sobre a estrutura foliar e o crescimento de plantas arbóreas. Revista Brasileira de Botânica, v.23, p.375-383, 2000.

SMITH, F.A.; HODGE, H.C. Airborne fluorides and man: part I. CRC Critical Review of Environment Control, v.8, p.397-372, 1979.

WEBER, J.A.; TINGEY, D.T.; ANDERSEN, C.P. Plant response to air pollution. In: WILKINSON, R.E. Plant-environment interactions. New York: Marcel Dekker, 1994. cap.12, p.357-389.

WEINSTEIN, L.H.; DAVISON, A.W. Native plant species suitable as bioindicators and biomonitors for airborne fluoride. Environmental Pollution, v.125, p.3-11, 2003.

WILSON, G.; AL-HAMDANI, S. Effects of chromium (VI) and humic substances on selected physiological responses of Azolla caroliniana. American Fern Journal, v.87, p.17-27, 1997.

ZHANG, J.; KIRKHAM, M.B. Lipid peroxidation in sorghum and sunflower seedlings as affected by ascorbic acid, benzoic acid, and propyl gallate. Journal of Plant Physiology, v.149, p.489-493, 1996.

Recebido em 24 de junho de 2004 e aprovado em 28 de fevereiro de 2005 